\title{
Classical stochastic systems with fast-switching environments: reduced master equations, their interpretation, and limits of validity
}

DOI:

10.1103/physreve.99.032121

\section{Document Version}

Accepted author manuscript

Link to publication record in Manchester Research Explorer

Citation for published version (APA):

Hufton, P., Lin, Y. T., \& Galla, T. (2019). Classical stochastic systems with fast-switching environments: reduced master equations, their interpretation, and limits of validity. Physical Review E: covering statistical, nonlinear, biological, and soft matter physics, 99, [032121]. https://doi.org/10.1103/physreve.99.032121

Published in:

Physical Review E: covering statistical, nonlinear, biological, and soft matter physics

\section{Citing this paper}

Please note that where the full-text provided on Manchester Research Explorer is the Author Accepted Manuscript or Proof version this may differ from the final Published version. If citing, it is advised that you check and use the publisher's definitive version.

\section{General rights}

Copyright and moral rights for the publications made accessible in the Research Explorer are retained by the authors and/or other copyright owners and it is a condition of accessing publications that users recognise and abide by the legal requirements associated with these rights.

\section{Takedown policy}

If you believe that this document breaches copyright please refer to the University of Manchester's Takedown Procedures [http://man.ac.uk/04Y6Bo] or contact uml.scholarlycommunications@manchester.ac.uk providing relevant details, so we can investigate your claim.

\section{OPEN ACCESS}




\title{
Classical stochastic systems with fast-switching environments: reduced master equations, their interpretation, and limits of validity
}

\author{
Peter G. Hufton, ${ }^{1}$ Yen Ting Lin, ${ }^{1,2}$ and Tobias Galla ${ }^{1}$ \\ ${ }^{1}$ Theoretical Physics, School of Physics and Astronomy, \\ The University of Manchester, Manchester M13 9PL, United Kingdom \\ ${ }^{2}$ Center for Nonlinear Studies and Theoretical and Biophysics Group, Theoretical Division, \\ Los Alamos National Laboratory, Los Alamos, New Mexico 87545, USA
}

(Dated: February 6, 2019)

\begin{abstract}
We study classical Markovian stochastic systems with discrete states, coupled to randomly switching external environments. For fast environmental processes we derive reduced dynamics for the system itself, focusing on corrections to the adiabatic limit of infinite time scale separation. We show that this can lead to master equations with bursting events. Negative transition 'rates' can result in the reduced master equation, leading to unphysical short-time behaviour. However, the reduced master equation can describe stationary states better than a leading-order adiabatic calculation, similar to what is known for Kramers-Moyal expansions in the context of the Pawula theorem. We provide an interpretation of the reduced dynamics in discrete time, and a criterion for the occurrence of negative rates for systems with two environmental states.
\end{abstract}

PACS numbers: 02.50.Ey Stochastic processes, 87.10.Mn Stochastic modeling, 87.18.Tt Noise in biological systems

\section{INTRODUCTION}

Physical and biological systems can never be fully isolated from their environment. This includes the dynamics of microbes in time-varying external conditions (e.g., antibiotic treatment) [1-4], or protein production in gene regulatory networks, influenced by the stochastic binding and unbinding of promoters [5-9]. Other examples can be found in models of evolutionary dynamics [10-13], the spread of diseases [14], and in ecology and population dynamics [15-18]. Many of models of these phenomena contain two types of randomness: one intrinsic to the system itself, and another generated by the noise in the environmental dynamics. The study of open quantum systems defines an entire area of research [19-21].

These examples share a common structure: there is the system proper and the environment, and a coupling between them; this interaction can act either in one way or in both directions. In such situations it is often not possible (or desirable) to track and analyse in detail the dynamics of the system and that of the environment. Instead the focus is on deriving reduced dynamics for the system itself, which in some way account for the influence of the environment on the system. Work on open quantum systems for example focuses on understanding the dynamics of reduced density matrices after integrating out the environment [19-21].

Existing work on open classical systems includes those described by stochastic differential equations (SDEs) coupled to continuous environments [10, 22-24], and deterministic models with discrete external noise [25-27]. A specific case of Brownian particles, subject to random external gating is considered in Ref. [28]. In chemical or biological systems the quasi-steady-state approximation or related adiabatic reduction techniques can be used to eliminate fast reactions $[29,30]$.
In this paper we consider open stochastic systems with discrete states. While some of our work is applicable more generally, we mostly focus on populations of interacting 'individuals'. We will often use the words 'system' and 'population' synonymously. Examples we have in mind are chemical reaction system with discrete molecules, or populations in biological systems, composed of members of different species. For a fixed environment, such a system is described by a (classical) master equation defined by the transition rates between its discrete states. These transitions are typically events in which particles are produced or removed from the population, or in which a particle of one type is converted into another type. In biological populations they can represent birth or death events. We are interested in cases in which such a population is coupled to an external environment, which also takes discrete states. The environmental states in turn affect the transition rates within the population.

Our aim is to study the reduced dynamics of such systems after the environmental dynamics are integrated out. In particular we focus on the limit in which the environmental dynamics are fast compared to those of the population, but where the separation of time scales is not infinite. We ask what the reduced dynamics looks like, if and how it can be interpreted, and how one might go about simulating it efficiently. In a companion paper (Ref. [31]) we build on these results, and carry out expansions of the reduced dynamics for weak intrinsic and environmental noise.

The remainder of the paper is organised as follows. In Sec. II we introduce the type of model we address, a classical stochastic system with discrete states coupled to an external environment, also with discrete states. We derive an effective master equation in the limit of fast time scales of the environmental switching; specifically, 
our analysis includes next-order corrections to the adiabatic limit of infinitely fast environments. In Sec. III we focus on the case of two environmental states, and use a simple example of a population with two species and two environmental states to illustrate the reduced dynamics. We show how negative transition 'rates' can arise in the reduced master equation. Sec. IV then focuses on the analysis of this reduced master equation in continuous time. We show that negative solutions can result at short time scales for certain initial condition. We comment on the origin of these unphysical transients, and demonstrate that the reduced master equation can nevertheless describe stationary distributions at long times with more accuracy than the dynamics in the adiabatic limit. In Sec. $\mathrm{V}$ we provide a physical interpretation of the reduced dynamics in discrete time. Specifically we show that a discrete-time process with reaction rates drawn from a Gaussian distribution at each time step can capture dynamical features of the full dynamics of system and environment. In Sec. VI finally we establish a criterion for the occurrence of negative rates for models in which the environmental dynamics has two states and is independent of the state of the population. We summarise our results in Sec. VII, and discuss their relevance and future lines of research which follow on from the work presented here.

\section{GENERAL DEFINITIONS AND REDUCED MASTER EQUATION}

\section{A. Model}

We focus on a classical system with discrete states, labelled $\ell$, which is coupled to an environment also taking discrete states, which we label $\sigma$. The system and the environment evolve in continuous time. The dynamics of the system itself depend on the current state of the environment. The environment in turn switches between its states, with transition rates which can depend on the state $\ell$ of the system. The combined dynamics of system and environment are then governed by the master equation

$$
\begin{aligned}
\frac{\mathrm{d}}{\mathrm{d} t} p(\ell, \sigma, t)= & \mathcal{M}_{\sigma} p(\ell, \sigma, t) \\
& +\lambda \sum_{\sigma^{\prime}} A_{\sigma^{\prime} \rightarrow \sigma}(\ell) p\left(\ell, \sigma^{\prime}, t\right)
\end{aligned}
$$

where $p(\ell, \sigma, t)$ is the joint probability of finding the system in state $\ell$ and the environment in state $\sigma$ at time $t$. The object $\mathcal{M}_{\sigma}$ is an operator, and determines how the state of the system can change when the environment is in state $\sigma$. More specifically, the effect of the operator can be written in the form

$$
\mathcal{M}_{\sigma} p(\ell, \sigma, t) \equiv \sum_{\ell^{\prime}} R_{\ell^{\prime} \rightarrow \ell}^{(\sigma)} p\left(\ell^{\prime}, \sigma, t\right) .
$$

The matrix element $R_{\ell^{\prime} \rightarrow \ell}^{(\sigma)}$ describes the rate at which the system transitions from state $\ell^{\prime}$ to state $\ell$ when the environment is in state $\sigma$. For a chemical reaction system, the types of allowed transitions are specified by the stoichiometric coefficients; together with associated reaction rates these determine the transition matrix. In the context of population dynamics the matrix $R_{\ell^{\prime} \rightarrow \ell}^{(\sigma)}$ is defined by the underlying birth and death processes (e.g., see Refs. [32, 33]).

The second term in Eq. (1), proportional to $\lambda$, characterises the environmental switching. The rate with which the environment transitions from state $\sigma$ to state $\sigma^{\prime}$ is $\lambda A_{\sigma \rightarrow \sigma^{\prime}}(\ell)$. In the most general setup, these can depend on the state $\ell$ of the system. We write $\lambda \mathbf{A}(\ell)$ for the corresponding transition matrix. The pre-factor $\lambda>0$ has been introduced to parametrise the time scale of the environment, relative to the internal dynamics of the population. To fix the diagonal elements of both transition matrices, we use the convention $R_{\ell \rightarrow \ell}^{(\sigma)}=-\sum_{\ell^{\prime} \neq \ell} R_{\ell \rightarrow \ell^{\prime}}^{(\sigma)}$, and $A_{\sigma \rightarrow \sigma}(\ell)=-\sum_{\sigma^{\prime} \neq \sigma} A_{\sigma \rightarrow \sigma^{\prime}}(\ell)$.

We introduce the notation $\Pi(\ell, t)=\sum_{\sigma} p(\ell, \sigma, t)$ for the marginal of the probability distribution after integrating out the environment. The general objective of this paper is to study the time evolution of this marginal distribution in the limit of fast-switching environments, $\lambda \gg 1$.

\section{B. Simplification in the adiabatic limit}

We first consider the so-called 'adiabatic' limit of infinitely fast environmental switching, $\lambda \rightarrow \infty$. In this limit and similar to [25] we find from Eq. (1)

$$
\sum_{\sigma^{\prime}} A_{\sigma^{\prime} \rightarrow \sigma}(\ell) p\left(\ell, \sigma^{\prime}, t\right)=0
$$

for all $\ell$. Writing the joint distribution $p(\ell, \sigma, t)$ in terms of the marginal $\Pi(\ell, t)$ and a conditional probability $\rho(\sigma \mid \ell, t)$, we have $p(\ell, \sigma, t)=\rho(\sigma \mid \ell, t) \Pi(\ell, t)$. Substituting this into Eq. (3) we find

$$
\sum_{\sigma^{\prime}} A_{\sigma^{\prime} \rightarrow \sigma}(\ell) \rho^{*}\left(\sigma^{\prime} \mid \ell\right)=0
$$

for all $\ell$, for the stationary distribution of the environment conditioned on the state of the system. We label this stationary distribution by an asterisk. In the adiabatic limit we then have

$$
p(\ell, \sigma, t)=\rho^{*}(\sigma \mid \ell) \Pi(\ell, t)
$$

We will use this relation as a starting point for further analysis; in the course of this analysis we also obtain the reduced dynamics for $\Pi(\ell, t)$ in the adiabatic limit. 


\section{Reduced master equation for large, but finite separation of time scales}

Our next aim is to derive reduced dynamics in the limit of fast environmental switching, but keeping the timescale separation finite (i.e., $\lambda$ large, but finite). Specifically, the objective is to derive a closed equation for the time-evolution of the distribution of states $\Pi(\ell, t)$. This is done by performing an expansion of the joint master equation for system and environment in powers of the time-scale separation $\lambda^{-1}$. We then retain the leading and sub-leading terms, and integrate out the environment. The algebraic steps are similar to those in Ref. [25], in which the authors work in the context of piecewisedeterministic Markov processes. We carry out the calculation starting from a system with discrete states $\ell$. As we will see below, this leads to interesting features of the reduced dynamics, not necessarily seen for continuous states.

To separate leading-order terms from sub-leading contributions we start with the decomposition

$$
p(\ell, \sigma, t)=\rho^{*}(\sigma \mid \ell) \Pi(\ell, t)+\frac{1}{\lambda} w_{\sigma}(\ell, t) .
$$

The term $w_{\sigma}(\ell, t)$ describes deviations from the adiabatic limit [Eq. (5)], due to a finite time scale of the environment. It includes corrections of order $\lambda^{-1}$ and higher, i.e. $w_{\sigma}(\ell, t)$ is in itself a power series. Because of normalisation, this ansatz requires $\sum_{\sigma} w_{\sigma}(\ell, t)=0$, for all $\ell$. We proceed by inserting Eq. (6) into Eq. (1), and obtain

$$
\begin{aligned}
& \rho^{*}(\sigma \mid \ell) \frac{\mathrm{d}}{\mathrm{d} t} \Pi(\ell, t)+\frac{1}{\lambda} \frac{\mathrm{d}}{\mathrm{d} t} w_{\sigma}(\ell, t) \\
& =\mathcal{M}_{\sigma}\left[\rho^{*}(\sigma \mid \ell) \Pi(\ell, t)\right]+\sum_{\sigma^{\prime}} A_{\sigma^{\prime} \rightarrow \sigma}(\ell) w_{\sigma^{\prime}}(\ell, t) \\
& \quad+\frac{1}{\lambda} \mathcal{M}_{\sigma} w_{\sigma}(\ell, t),
\end{aligned}
$$

where one further term has been eliminated using Eq. (4). Next, we sum over the environmental states $\sigma$ for each $\ell$. We find

$$
\frac{\mathrm{d}}{\mathrm{d} t} \Pi(\ell, t)=\sum_{\sigma} \mathcal{M}_{\sigma}\left[\rho^{*}(\sigma \mid \ell) \Pi(\ell, t)\right]+\frac{1}{\lambda} \sum_{\sigma} \mathcal{M}_{\sigma} w_{\sigma}(\ell, t) .
$$

Once the $w_{\sigma}(\ell, t)$ are expressed in terms of $\Pi(\ell, t)$, this equation describes the time-evolution of $\Pi(\ell, t)$, valid to sub-leading order in $\lambda^{-1}$.

To find the leading contribution to $w_{\sigma}(\ell, t)$ we collect the terms of order $(1 / \lambda)^{0}$ in Eq. (7),

$$
\begin{aligned}
\sum_{\sigma^{\prime}} A_{\sigma^{\prime} \rightarrow \sigma}(\ell) w_{\sigma^{\prime}}(\ell, t)= & \rho^{*}(\sigma \mid \ell) \sum_{\sigma^{\prime}} \mathcal{M}_{\sigma^{\prime}}\left[\rho^{*}\left(\sigma^{\prime} \mid \ell\right) \Pi(\ell, t)\right] \\
& -\mathcal{M}_{\sigma}\left[\rho^{*}(\sigma \mid \ell) \Pi(\ell, t)\right]
\end{aligned}
$$

where we have used Eq. (8) to further simplify the result. Effectively, we have disregarded terms of order $\lambda^{-1}$ in Eq. (7). This procedure indicates that the $w_{\sigma}(\ell, t)$ are to be obtained as the solution of Eq. (9), subject to $\sum_{\sigma} w_{\sigma}(\ell, t)=0$ for all $\ell$ and $t$. The truncation of higher order terms of leads to an error in Eq. (9) of order $\lambda^{-1}$. We note that in specific cases master equations for the system can be obtained in closed form without truncation (examples can be found in Refs. [34, 35]). These usually rely on specific properties of the model, such as linearity. Eqs. (8) and (9), while constituting an approximation to sub-leading order in $\lambda^{-1}$, hold more generally; we have not made significant restrictions on the dynamics of the system (i.e., on the operators $\mathcal{M}_{\sigma}$ ). For example, the approach can be used for population dynamics defined by birth and death processes, and with an arbitrary number of species. It can also be applied to spatially extended or networked systems.

\section{TWO ENVIRONMENTAL STATES: REDUCED MASTER EQUATION AND BASIC EXAMPLE}

\section{A. Environmental dynamics independent of the state of the system}

We now make a simplifying assumption, and consider the case in which the environmental switching dynamics are independent of the state of the population. That is to say, the transition rate matrix $A_{\sigma \rightarrow \sigma^{\prime}}$ does not depend on $\ell$. In this case, the stationary distribution of the environment in the adiabatic limit is independent of the state of population, i.e., $\rho^{*}(\sigma \mid \ell)=\rho_{\sigma}^{*}$. The more general case is discussed further in Appendix A and also in a further example in [31].

In this simplified case the dynamics in the adiabatic limit are given by

$$
\frac{\mathrm{d}}{\mathrm{d} t} \Pi(\ell, t)=\mathcal{M}_{\mathrm{avg}} \Pi(\ell, t),
$$

where $\mathcal{M}_{\text {avg }}=\sum_{\sigma^{\prime}} \rho_{\sigma^{\prime}}^{*} \mathcal{M}_{\sigma^{\prime}}$ is an effective, average operator. Equation (10) is obtained from Eq. (8) by sending $\lambda \rightarrow \infty$, and using $\rho^{*}(\sigma \mid \ell)=\rho_{\sigma}^{*}$. Equation (9), on the other hand, reduces to

$$
\sum_{\sigma^{\prime}} A_{\sigma^{\prime} \rightarrow \sigma} w_{\sigma^{\prime}}(\ell, t)=\rho_{\sigma}^{*}\left[\mathcal{M}_{\mathrm{avg}}-\mathcal{M}_{\sigma}\right] \Pi(\ell, t) .
$$

While the above procedure applies to an arbitrary number of discrete environmental states, it is useful to look at the case of two states, which we label $\sigma=0$ and $\sigma=1$. We then have $w_{0}(\ell, t)=-w_{1}(\ell, t)$ for all $\ell$ and $t$. To shorten the notation, we write $k_{0}$ and $k_{1}$ for the switching rates $A_{1 \rightarrow 0}$ and $A_{0 \rightarrow 1}$ respectively. In the adiabatic limit, the probabilities of finding the environment in each of its two states are then given by

$$
\rho_{0}^{*}=\frac{k_{0}}{k_{0}+k_{1}}, \quad \rho_{1}^{*}=\frac{k_{1}}{k_{0}+k_{1}} .
$$


From Eq. (11) one obtains

$$
w_{\sigma}(\ell, t)=\frac{k_{\sigma}}{\left(k_{0}+k_{1}\right)^{2}}\left[\mathcal{M}_{\sigma}-\mathcal{M}_{\mathrm{avg}}\right] \Pi(\ell, t)
$$

Substituting in Eq. (8) and simplifying, we arrive at

$$
\frac{\mathrm{d}}{\mathrm{d} t} \Pi(\ell, t)=\mathcal{M}_{\mathrm{avg}} \Pi(\ell, t)+\frac{1}{2} \frac{\theta^{2}}{\lambda}\left(\mathcal{M}_{0}-\mathcal{M}_{1}\right)^{2} \Pi(\ell, t),
$$

where

$$
\theta^{2}=\frac{2 k_{0} k_{1}}{\left(k_{0}+k_{1}\right)^{3}}
$$

For systems with two environmental states and with population-independent environmental switching, Eq. (14) is a general result approximating the dynamics in the limit of fast switching. It captures the timeevolution of $\Pi(\ell, t)$ up to and including sub-leading terms in $\lambda^{-1}$. We discuss limitations to its physical interpretation below. Despite these limitations we will refer to Eq. (14) (and its analogue for more complicated setups) as a reduced master equation. An expression similar to Eq. (14) was derived in Ref. [25] for systems with continuous states. We note that $\left(\mathcal{M}_{0}-\mathcal{M}_{1}\right)^{2}=$ $\left(\mathcal{M}_{0}^{2}-1\right)+\left(\mathcal{M}_{1}^{2}-1\right)-\left(\mathcal{M}_{0} \mathcal{M}_{1}-1\right)-\left(\mathcal{M}_{1} \mathcal{M}_{0}-1\right)$, indicating that Eq. (14) preserves total probability, i.e., $\frac{\mathrm{d}}{\mathrm{d} t} \sum_{\ell} \Pi(\ell, t)=0$.

\section{B. Example: population with two species}

\section{Reduced dynamics}

We next consider a specific example. This will help reveal a number of interesting features which can emerge in the reduced dynamics.

The example describes a population with two types of particles, labelled $A$ and $B$. Particles of either type are removed with constant per capita rates $\gamma$ and $\delta$, respectively, and are created with rates $\Omega \alpha_{\sigma}$ and $\Omega \beta_{\sigma}$. These production rates depend on the state of the environment, as indicated by the subscript. The population takes states $\ell=\left(n_{A}, n_{B}\right)$, where $n_{A}$ is the number of particles of type $A$, and $n_{B}$ the number of particles of type $B$. We then have operators

$$
\begin{aligned}
\mathcal{M}_{\sigma}= & \Omega \alpha_{\sigma}\left(\mathcal{E}_{A}^{-1}-1\right)+\gamma\left(\mathcal{E}_{A}-1\right) n_{A} \\
& +\Omega \beta_{\sigma}\left(\mathcal{E}_{B}^{-1}-1\right)+\delta\left(\mathcal{E}_{B}-1\right) n_{B},
\end{aligned}
$$

where $\mathcal{E}_{A} f\left(n_{A}, n_{B}\right)=f\left(n_{A}+1, n_{B}\right)$, and similarly for $\mathcal{E}_{B}$. The switching between environmental states is the same as in the previous section. Using Eq. (14) we find, to sub-leading order in $\lambda^{-1}$,

$$
\begin{aligned}
\frac{\mathrm{d}}{\mathrm{d} t} \Pi= & \gamma\left(\mathcal{E}_{A}-1\right) n_{A} \Pi+\delta\left(\mathcal{E}_{B}-1\right) n_{B} \Pi \\
& +\Omega \alpha_{\mathrm{eff}}\left(\mathcal{E}_{A}^{-1}-1\right) \Pi+\Omega \beta_{\mathrm{eff}}\left(\mathcal{E}_{B}^{-1}-1\right) \Pi \\
& +\frac{\Omega^{2} \theta^{2}}{2 \lambda}(\Delta \alpha)^{2}\left(\mathcal{E}_{A}^{-2}-1\right) \Pi \\
& +\frac{\Omega^{2} \theta^{2}}{2 \lambda}(\Delta \beta)^{2}\left(\mathcal{E}_{B}^{-2}-1\right) \Pi \\
& +\frac{\Omega^{2} \theta^{2}}{\lambda} \Delta \alpha \Delta \beta\left(\mathcal{E}_{A}^{-1} \mathcal{E}_{B}^{-1}-1\right) \Pi
\end{aligned}
$$

where $\Delta \alpha \equiv \alpha_{0}-\alpha_{1}$ and $\Delta \beta \equiv \beta_{0}-\beta_{1}$, and where

$$
\begin{aligned}
& \alpha_{\mathrm{eff}}=\alpha_{\mathrm{avg}}-\frac{\Omega \theta^{2}}{\lambda}(\Delta \alpha)^{2}-\frac{\Omega \theta^{2}}{\lambda} \Delta \alpha \Delta \beta \\
& \beta_{\mathrm{eff}}=\beta_{\mathrm{avg}}-\frac{\Omega \theta^{2}}{\lambda}(\Delta \beta)^{2}-\frac{\Omega \theta^{2}}{\lambda} \Delta \alpha \Delta \beta .
\end{aligned}
$$

The quantity $\alpha_{\text {avg }}$ is given by $\alpha_{\text {avg }}=\left(k_{0} \alpha_{0}+k_{1} \alpha_{1}\right) /\left(k_{0}+\right.$ $k_{1}$ ), and similar for $\beta_{\text {avg }}$. We have suppressed the explicit dependence of $\Pi$ on $n_{A}, n_{B}$ and $t$ to keep the notation compact.

We can interpret the reduced master equation as a set of reactions. The first two terms on the RHS of Eq. (17) describe particle removal, present already in the original model, and independent of the state of the environment. The terms in the second line are birth reactions, as appeared originally in the model. They describe the production of single particles of type $A$ or $B$. These reactions now occur with effective birth rates, indicated in Eq. (18). For a given set of model parameters these effective rates $\alpha_{\text {eff }}$ and $\beta_{\text {eff }}$ are non-negative, provided the switching is fast enough. Given that the reduced dynamics are derived in the limit $\lambda \gg 1$, we always assume that the time-scale separation $\lambda$ is large enough so that $\alpha_{\text {eff }}, \beta_{\text {eff }} \geq 0$.

The remaining terms in Eq. (17) represent reactions which are not present in the original model; they arise from the effects of integrating out the environment. These terms represent 'bursting' reactions; they describe events in which two particles of type $A$ are produced simultaneously, or two particles of type $B$, or one of either type. This is illustrated in Fig. 1. Panel (a) is a schematic showing the four states that the population can reach from a given state in the next event in the original model. Panel (b) shows that the reduced dynamics allow three additional destinations (indicated by grey dashed arrows). The rates of the first two bursting reactions in Eq. (17) are proportional to $(\Delta \alpha)^{2}$ and $(\Delta \beta)^{2}$, and are always positive [lines three and four on the right-hand side of Eq. (17)]. The rate of the third bursting reaction [last term on RHS of Eq. (17)] is positive only if $\Delta \alpha$ and $\Delta \beta$ have the same sign. We therefore distinguish between the cases $\Delta \alpha \Delta \beta>0$ and $\Delta \alpha \Delta \beta<0$. 
(a)

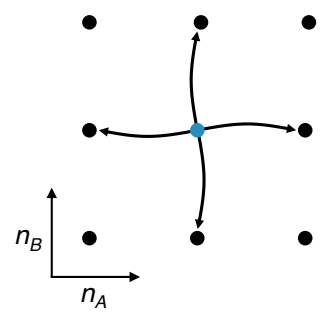

(b)

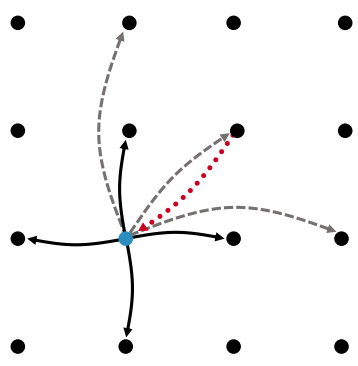

FIG. 1. Illustration of the possible reactions for (a) the model described by equations (16), and (b) the approximation to the model described by Eq. (17). In the original model the next event can take the population from $\left(n_{A}, n_{B}\right)$ to four possible destinations: $\left(n_{A} \pm 1, n_{B}\right),\left(n_{A}, n_{B} \pm 1\right)$. The bursting reactions in the reduced model lead to further states which can be reached, indicated by grey dashed arrows; these are $\left(n_{A}+2, n_{B}\right),\left(n_{A}, n_{B}+2\right),\left(n_{A}+1, n_{B}+1\right)$. For certain choices of parameters the transition to $\left(n_{A}+1, n_{B}+1\right)$ can have a negative 'rate'. In this case the flow of probability is from $\left(n_{A}+1, n_{B}+1\right)$ to $\left(n_{A}, n_{B}\right)$ as indicated by the red dotted arrow; see Sec. IV A for details.

\section{Positive correlation between the species}

In the case $\Delta \alpha \Delta \beta>0$, the correlations between $n_{A}$ and $n_{B}$ are positive. There is one state of the environment which favours both species, i.e., they each have a higher birth rate in this environmental state than in the other. All rates in Eq. (17) are positive (provided $\lambda$ is sufficiently large, so that $\alpha_{\text {eff }}, \beta_{\text {eff }} \geq 0$ ). There is then a clear and unique way of interpreting this master equation as a continuous-time Markov process. The events described by the various terms are as above: single deaths, single births, and bursting reactions in which two particles are produced. The notion of sample paths is well-defined; they can be generated using the standard Gillespie algorithm [36, 37].

Some support for the validity of the reduced master equation in describing stationary states is given in Fig. 2, panels (a)-(c). In panel (a) we show the stationary distribution obtained from numerically integrating the full master equation Eq. (1), i.e., from the full dynamics of population and environment. This is for the case $\Delta \alpha \Delta \beta>0$. Panel (b) shows the corresponding distribution from numerical integration of the reduced master equation (17). In panel (c) we have taken the adiabatic limit $\lambda \rightarrow \infty$. In each case the numerical integration is carried out using a Runge-Kutta scheme (RK4). The reduced dynamics capture the correlations between $n_{A}, n_{B}$ in the original model; this correlation is no longer seen in the adiabatic approximation. Panel (d) shows the marginal distribution for the quantity $n_{A}+n_{B}$ to allow better comparison.

These observations lead to the following physical picture. In the case of infinitely fast environments (adiabatic limit) the noise from then environmental process is entirely neglected, resulting in a narrower stationary dis-

tribution [Fig. 2(d)]. In addition, our analysis shows the environmental process introduces a positive correlation between $n_{A}$ and $n_{B}$. This correlation is lost in the limit of infinitely fast environmental switching.

\section{Anti-correlations and negative transition rates}

When $\Delta \alpha$ and $\Delta \beta$ have opposite signs, the interpretation of Eq. (17) presents an interesting feature. In this situation the (pseudo-) rate of the last reaction $\left(\Omega^{2} \theta^{2} / \lambda\right) \Delta \alpha \Delta \beta$ is negative, irrespective of the value of $\lambda$. The interpretation of this term is then not clear $a$ priori, and Eq. (17) is not a master equation in the usual sense. We will nevertheless refer to it as the reduced master equation, quotation marks or a prefix 'pseudo-' are implied. Similarly, we will continue to speak of rates, even if these are negative. From Eq. (17) it is clear that negative rates of this type are present in this example if and only if $\Delta \alpha \Delta \beta<0$.

We discuss the interpretation and limitations of master equation with negative rates in the next Section.

\section{INTERPRETATION OF NEGATIVE RATES: CONTINUOUS TIME}

\section{A. Flow of probability and 'renormalised' reaction rates}

In order to better understand a master equation with negative rates, we focus on a pair of states, which we label $\ell$ and $\ell^{\prime}$, and on a single reaction of type $\ell \rightarrow \ell^{\prime}$ occurring with a rate $R_{\ell \rightarrow \ell^{\prime}}$. In the specific example above one would have $\ell=\left(n_{A}, n_{B}\right)$ and $\ell^{\prime}=\left(n_{A}+1, n_{B}+1\right)$. The corresponding terms in the master equation are then

$$
\begin{aligned}
\frac{\mathrm{d}}{\mathrm{d} t} \Pi(\ell, t) & =-R_{\ell \rightarrow \ell^{\prime}} \Pi(\ell, t), \\
\frac{\mathrm{d}}{\mathrm{d} t} \Pi\left(\ell^{\prime}, t\right) & =R_{\ell \rightarrow \ell^{\prime}} \Pi(\ell, t) .
\end{aligned}
$$

In conventional cases the rate is positive, $R_{\ell \rightarrow \ell^{\prime}}>0$. The master equation then describes a non-negative probability flow $R_{\ell \rightarrow \ell^{\prime}} \Pi(\ell)$ from $\ell$ to $\ell^{\prime}$ (we suppress the time dependence of $\Pi(\ell)$ for convenience).

For $R_{\ell \rightarrow \ell^{\prime}}<0$, the flow of probability per unit time in Eqs. (19) is $\left|R_{\ell \rightarrow \ell^{\prime}}\right| \Pi(\ell) \geq 0$ from $\ell^{\prime}$ to $\ell$. This is different from situation in conventional Markovian systems. The flow is directed from $\ell^{\prime}$ to $\ell$, but proportional to the probability already present at $\ell$. This is illustrated for the two-species model by the red arrow from $\left(n_{A}+1, n_{B}+1\right)$ to $\left(n_{A}, b_{N}\right)$ in Fig. 1. Furthermore, the magnitude of this flow does not depend on $\Pi\left(\ell^{\prime}\right)$. Instead it is proportional to $\Pi(\ell)$, the probability of the state toward which the flow is directed. In making this argument, we have assumed $\Pi(\ell) \geq 0$. This assumption is not always justified in master equations with negative rates, see below. However the above argument holds more generally: a negative 

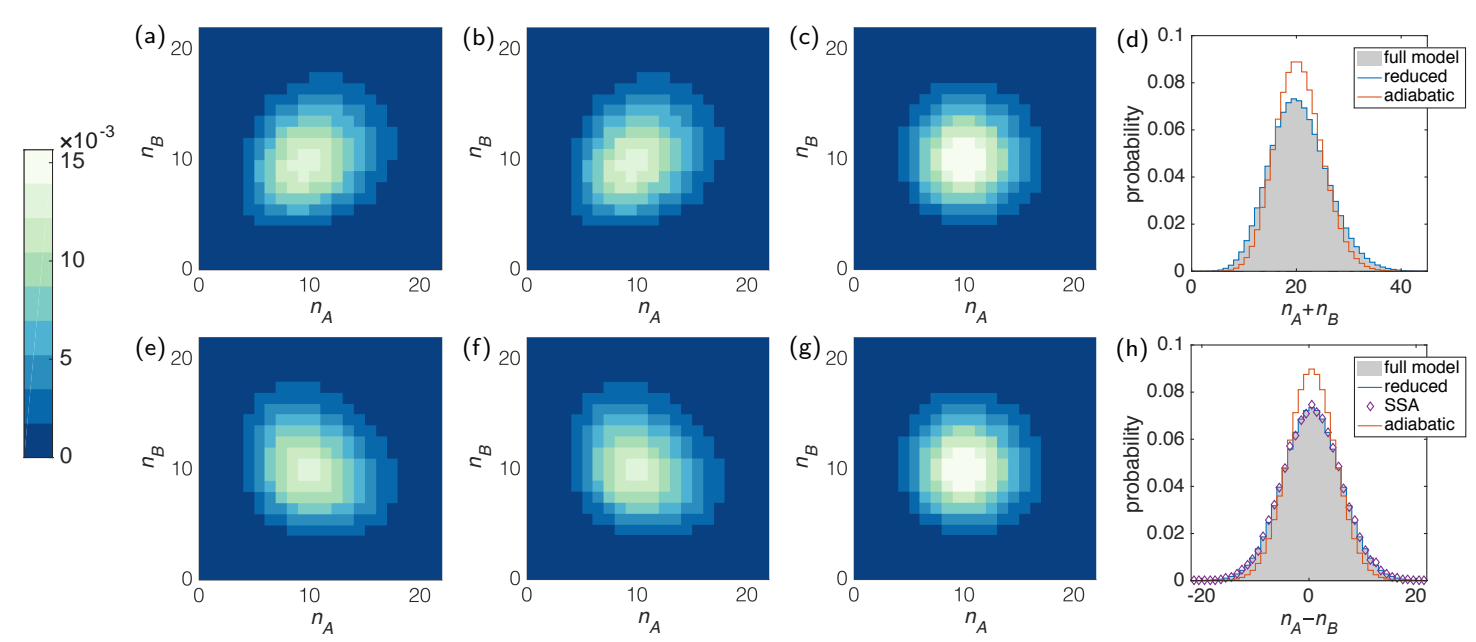

FIG. 2. Stationary distribution of the model defined in Sec. III B [see Eqs. (16)]. The upper panels are for $\Delta \alpha \Delta \beta>0$, the lower row for $\Delta \alpha \Delta \beta<0$. The distributions are obtained by numerical integration of: (a,e) the full master equation with explicit environment, (b,f) the reduced master Eq. (17), and $(\mathrm{c}, \mathrm{g})$ the adiabatic approximation; (d) shows the marginal distribution of $n_{A}+n_{B}$; panel (h) shows $n_{A}-n_{B}$. Markers labelled 'SSA' in panel (h) are from the stochastic simulation algorithm (SSA) described in Appendix B 1. Parameters are $\alpha_{0}=0, \alpha_{1}=1, \beta_{0}=0, \beta_{1}=1$ in the upper row, and $\alpha_{0}=0, \alpha_{1}=1, \beta_{0}=1, \beta_{1}=0$ in the lower row. Remaining parameters are $\Omega=20, \lambda=20, k_{0}=k_{1}=1$.

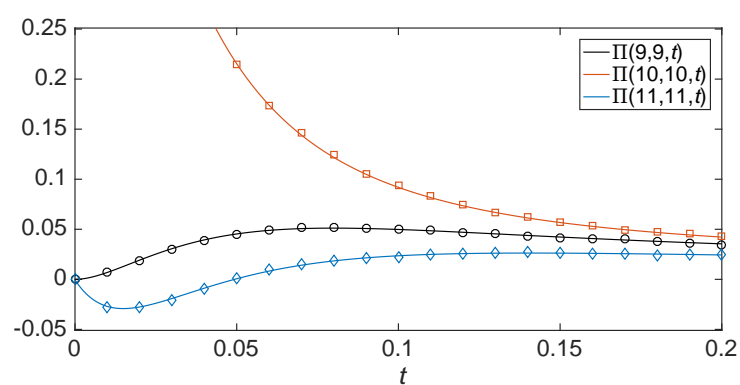

FIG. 3. Time evolution of several entries $\Pi\left(n_{A}, n_{B}, t\right)$ for the example defined in Sec. IIIB. The solid lines show results from integrating the reduced master equation (17), starting from a delta-distribution $\Pi\left(n_{A}, n_{B}, t=0\right)=\delta_{n_{A}, 10} \delta_{n_{B}, 10}$. Markers are from the numerical simulation scheme described in Appendix B 2. Model parameters are $\alpha_{0}=0, \alpha_{1}=1, \beta_{0}=$ $1, \beta_{1}=0, \Omega=20, \lambda=20, k_{0}=k_{1}=1$.

value of $R_{\ell \rightarrow \ell^{\prime}} \Pi(\ell)$ indicates a positive probability flux $\left|R_{\ell \rightarrow \ell^{\prime}} \Pi(\ell)\right|$ from $\ell^{\prime}$ to $\ell$.

An approach to renormalising master equations with negative rates has been proposed in Refs. [38, 39] in the context of open quantum systems. We illustrate this using Eqs. (19), assuming again $R_{\ell \rightarrow \ell^{\prime}}<0$. For $\Pi\left(\ell^{\prime}\right)>0$ one defines the renormalised transition rate

$$
T_{\ell^{\prime} \rightarrow \ell}(t) \equiv \frac{\Pi(\ell, t)}{\Pi\left(\ell^{\prime}, t\right)}\left|R_{\ell \rightarrow \ell^{\prime}}\right|
$$

The master equation (19) can be then written as

$$
\begin{aligned}
\frac{\mathrm{d}}{\mathrm{d} t} \Pi(\ell, t) & =T_{\ell^{\prime} \rightarrow \ell}(t) \Pi\left(\ell^{\prime}, t\right), \\
\frac{\mathrm{d}}{\mathrm{d} t} \Pi\left(\ell^{\prime}, t\right) & =-T_{\ell^{\prime} \rightarrow \ell}(t) \Pi\left(\ell^{\prime}, t\right) .
\end{aligned}
$$

Equations (21), then, resemble a more traditional master equation, and $T_{\ell^{\prime} \rightarrow \ell}$ is the rate for transitions from $\ell^{\prime}$ to $\ell$. However, this rate depends on the probability distribution $\Pi$, in particular $T_{\ell^{\prime} \rightarrow \ell}$ is a function of $\Pi(\ell)$. This indicates non-Markovian properties [20, 38, 39]. The renormalised reaction rates can be used to adapt Gillespie's simulation algorithm for the generation of sample paths of stochastic systems [36, 37]. We describe these adaptations in Appendix B 1. We have tested the resulting algorithm on the two-species example with $\Delta \alpha \Delta \beta<0$, and as seen in Fig. 2(h), it captures the anti-correlation of $n_{A}$ and $n_{B}$ in the stationary distribution. However, the algorithm does not reproduce all dynamical features of the original dynamics of system and environment (see Appendix B 1). A separate sampling algorithm is described in Appendix B 2. This method simulates the solution of reduced master equations (including those with negative rates), but it does not attempt to generate sample paths.

\section{B. Lack of positivity in initial transients}

The reduced master equation (8) preserves overall probability, in the sense that $\sum_{\ell} \frac{\mathrm{d} \Pi(\ell, t)}{\mathrm{d} t}=0$. However, if a negative rate is present, one can always find initial conditions so that negative solutions result at short times. Assume for example that $R_{\ell \rightarrow \ell^{\prime}}<0$ for a particular pair of states $\ell$ and $\ell^{\prime}$. We now choose initial condition $\Pi(\ell, t=0)=1$, and vanishing initial probability for all other states. From Eq. (19) one then sees that $\frac{\mathrm{d} \Pi(\ell, 0)}{\mathrm{d} t}=-R_{\ell \rightarrow \ell^{\prime}}>0$, and $\frac{\mathrm{d} \Pi\left(\ell^{\prime}, 0\right)}{\mathrm{d} t}=R_{\ell \rightarrow \ell^{\prime}}<0$. Thus, $\Pi\left(\ell^{\prime}, t\right)$ will go negative.

We have verified this by numerically integrating the reduced master equation (17). For example, if the ini- 


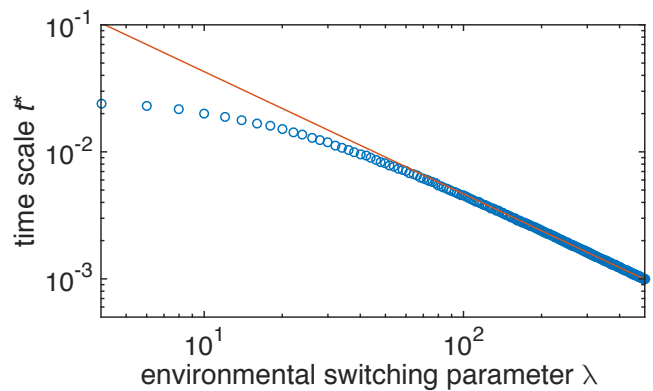

FIG. 4. Time scale $t^{*}$ over which negative probabilities are accumulated. Specifically, $t^{*}$ is the time at which the sum of all negative entries in $\Pi$ has maximal magnitude; data is from numerical integration of Eq. (17). The solid line is a guide and corresponds to $t^{*} \propto 1 / \lambda$. Parameters and initial condition are the same as Fig. 3.

tial condition is chosen as a delta-peak concentrated on one state $\ell=\left(n_{A}, n_{B}\right)$, the numerical solution for $\Pi\left(n_{A}+1, n_{B}+1\right)$ is negative for a limited time as shown in Fig. 3. We analyse this further in Fig. 4, where we show the duration $t^{*}$ of the initial transient in which negative probabilities are accumulated. The data suggests that this time window is limited to a duration of order $\lambda^{-1}$.

It is not surprising that Eq. (17) should become unphysical on short time scales. The typical time between switches of the environmental state is of order $\lambda^{-1}$, and the reduced dynamics were derived by integrating out the fast environmental dynamics. We cannot expect Eq. (17) to resolve the physics of the problem on time scales shorter than order $\lambda^{-1}$, as then the detailed mechanics of the environment become important. We note that negative transients have been observed before in reduced dynamics for open classical and quantum systems [40-43].

We have verified that the appearance of transient negative solutions can be cured by first integrating the full master equation describing the population and the environment for a short period of time, and then subsequently changing to the reduced master equation (17). Alternatively, the reduced dynamics can be started from 'slipped' initial conditions [40, 42].

Parallels can be drawn between the occurrence of negative transients in reduced master equations and the well-known Pawula theorem for Kramers-Moyal expansions $[44,45]$. This theorem shows that carrying out a Kramers-Moyal expansion on a discrete stochastic system only produces physical results for the dynamics if the expansion truncated after terms of order one (resulting in a Liouville equation describing the deterministic flow in the limit of infinite populations), or of order two (resulting in a Fokker-Planck equation describing the diffusion approximation of the individual-based model). Truncation at any higher order can lead to negative transients. This does not, however, imply that such expansions are of no use. On the contrary, outside the initial transient a truncation to order $n \geq 3$ of the Kramers-Moyal expan- sion can produce better agreement with the probability distribution of the system that is being approximated than the traditional Fokker-Planck truncation $(n=2)$, see Ref. [45] for details and examples. Even though the expansion may lead to unphysical results at short times, it may still be valuable at long times, for example to calculate stationary distributions.

We have tested this for the model with two types of particles defined in Sec. III B. The stationary distribution obtained from numerical integration of the reduced master equation (17) for $\Delta \alpha \Delta \beta<0$ captures the negative correlation of $n_{A}$ and $n_{B}$ in the original dynamics. This can be seen in Fig. 2(e) and (f). Working in the adiabatic limit, however, one finds significant deviations [panels (g) and (h)].

\section{INTERPRETATION IN DISCRETE TIME}

The results of the previous Section indicate that the reduced master equation containing negative transition rates does not have a physical interpretation at short time scales. Solutions can go negative at short times, and as a consequence reduced master equations with negative rates do not describe the statistics of a continuous-time stochastic processes. In this Section we show that a valid physical reduced dynamics can be formulated for discrete time steps. To do this we look the full dynamics of system and environment in discrete time, and provide an interpretation of the reduced dynamics at the level of discrete-time sample paths.

\section{A. Effective time-averaged reaction rates}

We focus again on the two-species example given in Sec. III. An interpretation of the terms in Eq. (17) can be obtained by looking at one sample path of the full model (population and environment) for a time interval $I \equiv\left[t_{0}, t_{0}+\Delta t\right]$. We focus on the birth reactions. If the production rate $\Omega \alpha$ of particles of type $A$ were constant in time, the number of birth events in the interval would be a Poissonian random variable with parameter $\Omega \alpha \Delta t$, and similarly for particles of type $B$ (see also Ref. [46]). In the present model, the production rates are not constant as they depend on the time-dependent state of the environment. For a given trajectory of the environment we introduce the quantity

$$
\bar{\alpha}=\frac{1}{\Delta t} \int_{t_{0}}^{t_{0}+\Delta t} \mathrm{~d} t^{\prime} \alpha_{\sigma\left(t^{\prime}\right)}
$$

and a similar definition for $\bar{\beta}$; the quantities $\Omega \bar{\alpha}$ and $\Omega \bar{\beta}$ are time-averaged production rates in the time interval $I$.

We note that $\bar{\alpha}$ and $\bar{\beta}$ are random variables when $\Delta t$ is finite, as they depend on the random path of the environment, $\sigma\left(t^{\prime}\right), t^{\prime} \in I$. We suppress the dependence 
of $\bar{\alpha}$ and $\bar{\beta}$ on $t_{0}$ in our notation. The quantities $\bar{\alpha}$ and $\bar{\beta}$ will in general be correlated, as they derive from the same realisation of the environment. The main principle of the calculation that follows is to approximate $\bar{\alpha}$ and $\bar{\beta}$ as correlated Gaussian random variables, while capturing their first and second moments. This Gaussian approximation is justified provided that there is a large number of switches of the environment during the time interval $I$, i.e., when $\lambda \Delta t \gg 1$. The number of production events of particles of type $A$ in $I$ can then be expected to be Poissonian with parameter $\Omega \bar{\alpha} \Delta t$, and similarly for $B$.

\section{B. Averaging out the environmental process}

Correlations of the environmental process decay on time scales proportional to $\lambda^{-1}$. This means that the environment is in its stationary distribution, except for a short period of order $\lambda^{-1}$ at the beginning. For $\lambda \Delta t \gg 1$ this period constitutes a negligibly small fraction of the time interval, and the distribution of $\sigma\left(t^{\prime}\right)$ can hence be assumed to be the stationary one at all times $t^{\prime}$ during the interval. Writing $\langle\ldots\rangle$ for averages over the environmental process we have $\langle\bar{\alpha}\rangle=\alpha_{\text {avg }}$ and $\langle\bar{\beta}\rangle=\beta_{\text {avg }}$.

For the second moment of $\bar{\alpha}$ we find

$$
\begin{aligned}
\left\langle\bar{\alpha}^{2}\right\rangle= & (\Delta t)^{-2} \int_{I} \int_{I} \mathrm{~d} t \mathrm{~d} t^{\prime}\left\langle\alpha_{\sigma(t)} \alpha_{\sigma\left(t^{\prime}\right)}\right\rangle, \\
= & (\Delta t)^{-2} \sum_{\sigma \sigma^{\prime}} \alpha_{\sigma} \alpha_{\sigma^{\prime}} \\
& \times \int_{I} \int_{I} \mathrm{~d} t \mathrm{~d} t^{\prime} \rho\left[\sigma, \min \left(t, t^{\prime}\right)\right] \rho\left(\sigma^{\prime},\left|t-t^{\prime}\right| \mid \sigma\right),
\end{aligned}
$$

where $\rho\left[\sigma, \min \left(t, t^{\prime}\right)\right]$ is the probability distribution of $\sigma$ at the earlier of the two times $t$ and $t^{\prime}$. It is given by the stationary distribution of the environment, $\rho\left[\sigma, \min \left(t, t^{\prime}\right)\right]=$ $\rho_{\sigma}^{*}$, with $\rho_{\sigma}^{*}$ as in Eq. (12). The notation $\rho\left(\sigma^{\prime}, \tau \mid \sigma\right)$ in Eq. (23) indicates the probability of finding the environment in state $\sigma^{\prime}$ if $\tau$ units of time earlier it was in state $\sigma(\tau>0)$. These can be obtained straightforwardly from the asymmetric telegraph process for the environment, $\rho(0, \tau \mid 0)=\rho_{0}^{*}\left[1+\frac{k_{1}}{k_{0}} e^{-\lambda\left(k_{0}+k_{1}\right) \tau}\right]$, and $\rho(0, \tau \mid 1)=\rho_{0}^{*}\left[1-e^{-\lambda\left(k_{0}+k_{1}\right) \tau}\right]$. Using this in Eq. $(23)$ we find

$$
\begin{aligned}
\left\langle\bar{\alpha}^{2}\right\rangle= & \alpha_{\text {avg }}^{2}+\left[\frac{2}{\lambda\left(k_{0}+k_{1}\right) \Delta t}+\frac{2}{\lambda^{2}\left(k_{0}+k_{1}\right)^{2} \Delta t^{2}}\right. \\
& \left.\times\left(e^{-\lambda\left(k_{0}+k_{1}\right) \Delta t}-1\right)\right] \frac{k_{0} k_{1}}{\left(k_{0}+k_{1}\right)^{2}}\left(\alpha_{0}-\alpha_{1}\right)^{2} .
\end{aligned}
$$

For $\lambda \Delta t \gg 1$ the first term in the square bracket dominates relative to the second, so we can approximate

$$
\left\langle\bar{\alpha}^{2}\right\rangle-\alpha_{\text {avg }}^{2} \approx \frac{\theta^{2}}{\lambda \Delta t}(\Delta \alpha)^{2}
$$

with $\theta^{2}=2 k_{0} k_{1} /\left(k_{0}+k_{1}\right)^{3}$ as before [see Eq. (15)]. Following similar steps one finds

$$
\begin{aligned}
\left\langle\bar{\beta}^{2}\right\rangle-\beta_{\mathrm{avg}}^{2} & \approx \frac{\theta^{2}}{\lambda \Delta t}(\Delta \beta)^{2}, \\
\langle\bar{\alpha} \bar{\beta}\rangle-\alpha_{\mathrm{avg}} \beta_{\mathrm{avg}} & \approx \frac{\theta^{2}}{\lambda \Delta t} \Delta \alpha \Delta \beta .
\end{aligned}
$$

We therefore approximate the joint probability distribution of $\bar{\alpha}$ and $\bar{\beta}$ in the fast switching limit as a bivariate normal distribution with these parameters.

\section{Resulting event statistics}

The probability that exactly $m_{A}$ production events for species $A$ occur during the time interval $\Delta t$, and $m_{B}$ for species $B$, is given by

$$
\mathrm{P}\left(m_{A}, m_{B}\right)=\left\langle e^{-\Delta t \Omega(\bar{\alpha}+\bar{\beta})} \frac{(\Delta t \Omega \bar{\alpha})^{m_{A}}}{m_{A} !} \frac{(\Delta t \Omega \bar{\beta})^{m_{B}}}{m_{B} !}\right\rangle_{\bar{\alpha}, \bar{\beta}},
$$

resulting from Poissonian statistics for given $\bar{\alpha}, \bar{\beta}$, subsequently averaged over the Gaussian distribution for $\bar{\alpha}$ and $\bar{\beta}$ (this average is indicated as $\langle\ldots\rangle_{\bar{\alpha}}, \bar{\beta}$ ). Expanding in powers of $\Delta t$, and carrying out the Gaussian average we find

$$
\begin{aligned}
\mathrm{P}\left(m_{A}=1, m_{B}=0\right)= & \Delta t \Omega\left[\alpha_{\text {avg }}-\frac{\Omega \theta^{2}}{\lambda}(\Delta \alpha)^{2}-\frac{\Omega \theta^{2}}{\lambda} \Delta \alpha \Delta \beta\right] \\
& -\Delta t^{2} \Omega^{2}\left(\alpha_{\text {avg }}^{2}+\beta_{\text {avg }}^{2}\right), \\
\mathrm{P}\left(m_{A}=0, m_{B}=1\right)= & \Delta t \Omega\left[\beta_{\text {avg }}-\frac{\Omega \theta^{2}}{\lambda}(\Delta \beta)^{2}-\frac{\Omega \theta^{2}}{\lambda} \Delta \alpha \Delta \beta\right] \\
& -\Delta t^{2} \Omega^{2}\left(\alpha_{\text {avg }}^{2}+\beta_{\text {avg }}^{2}\right), \\
\mathrm{P}\left(m_{A}=2, m_{B}=0\right)= & \frac{1}{2} \Delta t \frac{\Omega^{2} \theta^{2}}{\lambda}(\Delta \alpha)^{2}+\frac{1}{2} \Delta t^{2} \Omega^{2} \alpha_{\text {avg }}^{2}, \\
\mathrm{P}\left(m_{A}=0, m_{B}=2\right)= & \frac{1}{2} \Delta t \frac{\Omega \theta^{2}}{\lambda}(\Delta \beta)^{2}+\frac{1}{2} \Delta t^{2} \Omega^{2} \beta_{\text {avg }}^{2}, \\
\mathrm{P}\left(m_{A}=1, m_{B}=1\right)= & \frac{\Omega^{2} \theta^{2}}{\lambda} \Delta t \Delta \alpha \Delta \beta+\Delta t^{2} \Omega^{2} \alpha_{\text {avg }} \beta_{\text {avg }},
\end{aligned}
$$

where we have ignored higher-order terms (those which go like $\Delta t^{3}$ or $\left.\Delta t^{2} / \lambda\right)$. Larger numbers of production events $\left(m_{A}+m_{B} \geq 3\right)$ do not contribute at this order.

It is tempting to consider the limit of infinitesimally small $\Delta t$, and to use the first-order terms in $\Delta t$ in Eq. (27) to construct reaction rates. If one does so, one recovers the rates exactly as they appear in the reduced master equation (17); for example one would infer a rate of $\frac{1}{2}\left(\Omega^{2} \theta^{2} / \lambda\right)(\Delta \alpha)^{2}$ for events in which two particles of type $A$ are produced and none of type $B$ $\left(m_{A}=2, m_{B}=0\right)$. The rate of an event in which one $A$ and one $B$ are produced simultaneously would be $\left(\Omega^{2} \theta^{2} / \lambda\right) \Delta \alpha \Delta \beta$, which is negative if $\Delta \alpha \Delta \beta<0$.

However, taking the limit $\Delta t \rightarrow 0$ at fixed $\lambda$ is not compatible with the assumption that a large number of environmental switching events occur in a given timestep, i.e., $\lambda \Delta t \gg 1$. 
To illustrate this we carried out simulations of the full model of population and environment, and measured how many birth events of either particle type occur in a typical time interval of length $\Delta t$. Specifically we focus on the probability $P\left(m_{A}=1, m_{B}=1\right)$ of seeing exactly one birth event of type $A$ and one birth event of type $B$ during such a time interval; note that in the full model these births occur in two separate events. Results are shown in Fig. 5. The solid lines show the predictions of Eqs. (27), results from simulations of the full model are shown as markers. We first notice that simulations deviate from the results of Eqs. (27) at large values of $\Delta t$. This is to be expected as Eqs. (27) are derived neglecting higher-order terms in $\Delta t$. Simulations and the above expressions agree to good accuracy at intermediate values of the time step; we write $\Delta t_{*}$ for the lower end of this range, and $\Delta t^{*}$ for the upper end. As seen in Fig. 5, the lower threshold $\Delta t_{*}$ decreases as the switching of the environment becomes faster (i.e., $\lambda$ is increased). The reduction of the threshold is in-line with the requirement $\lambda \Delta t \gg 1$ for the theoretical analysis above.

When the predictions of Eqs. (27) for $P\left(m_{A}=1, m_{B}=\right.$ 1) match simulations of the full model they are largely determined by the term of order $\Delta t^{2}$, see again Fig. 5 (the slope of the simulation data in the log-log plot is then approximately two as indicated by the dashed lines). This term, $\Delta t^{2} \Omega^{2} \alpha_{\text {avg }} \beta_{\text {avg }}$, is positive, irrespective of the sign of $\Delta \alpha \Delta \beta$. At low values of $\Delta t \lesssim \Delta t_{*}$, we observe systematic deviations between simulations of the full model and the expressions in Eqs. (27). For the case $\Delta \alpha \Delta \beta<0$ it is obvious that this must occur: at small $\Delta t$, Eqs. (27) predict $\mathrm{P}\left(m_{A}=1, m_{B}=1\right) \approx\left(\Omega^{2} \theta^{2} / \lambda\right) \Delta t \Delta \alpha \Delta \beta<0$, whereas $P\left(m_{A}=1, m_{B}=1\right)$ is non-negative in simulations by definition. Deviations at small time steps are also seen when $\Delta \alpha \Delta \beta>0$, see the left-hand panel of Fig. 5. The expression in Eqs. (27) shows a cross-over to linear scaling in $\Delta t$, whereas simulation results scale approximately as $\Delta t^{2}$.

The construction in this Section confirms the limitations of the reduced master equation when negative rates are present. Some of the expressions in Eq. (27) become unphysical at short time scales, just like the transient solutions of the reduced master equation. This is due to the expansion used when going from Eq. (24) to Eq. (25), where we explicitly assumed $\lambda \Delta t \gg 1$. At the same time, the discrete-time approach reveals that a physically meaningful reduced process can be defined provided $\Delta t$ is not too small. We use this to describe a concrete algorithm for the simulation of discrete-time sample paths in the next Section.

\section{Simulation procedure for discrete-time sample paths}

The analysis of the previous section is based on a discretisation of time into intervals of length $\Delta t$. In the limit of fast switching of the environment it then assumes that
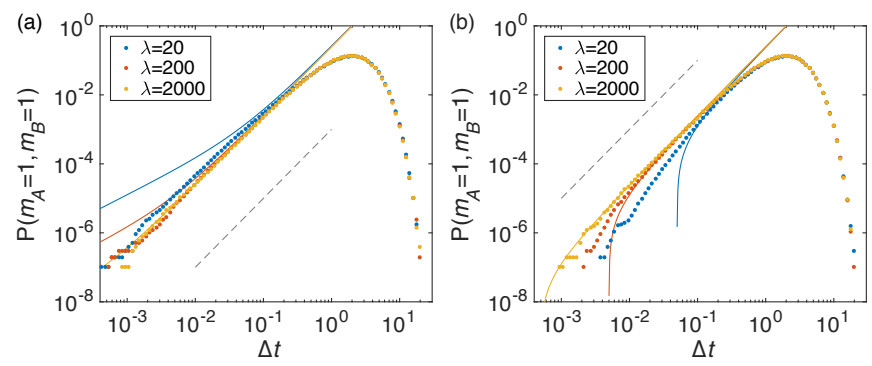

FIG. 5. Probability of seeing $m_{A}=1, m_{B}=1$ in a given time interval of duration $\Delta t$. Circles show the results of simulation of the full model (population and environment); full lines show Eq. (27). The dashed line shows a slope of 2 for comparison. Data is shown for different values of $\lambda$, all other model parameters are as in the earlier figures. (a) $\Delta \alpha \Delta \beta>0$, (b) $\Delta \alpha \Delta \beta<0$.

the time-averaged birth rates $\Omega \bar{\alpha}$ and $\Omega \bar{\beta}$ are Gaussian random variables with statistics given in Eqs. (25). We will now use this interpretation to define an algorithm with which to approximate sample paths of the full model in discrete time. We note that $\bar{\alpha}$ and $\bar{\beta}$ can take negative values in this Gaussian approximation. This issue arises irrespective of the sign of $\Delta \alpha \Delta \beta$ and is separate from the problem of negative rates in the reduced master equation. The probability for $\bar{\alpha}$ and/or $\bar{\beta}$ to be negative is exponentially suppressed in $\lambda \Delta t$, as the mean of the Gaussian distribution, $\left(\alpha_{\text {avg }}, \beta_{\text {avg }}\right)$, does not depend on $\lambda$ or $\Delta t$, and the covariance matrix is of order $(\lambda \Delta t)^{-1}$ [Eq. (25)]. As the switching of the environment becomes faster the distributions of $\bar{\alpha}$ and $\bar{\beta}$ become increasingly peaked around their mean. For the purposes of the numerical scheme we truncate the distribution at zero.

The algorithm uses ideas from the $\tau$-leaping variant of the Gillespie algorithm [46], and proceeds as follows:

1. Assume the simulation has reached time $t$ and that the current particle numbers are $n_{A}$ and $n_{B}$. Draw correlated Gaussian random numbers $\bar{\alpha}$ and $\bar{\beta}$, from a distribution with $\langle\bar{\alpha}\rangle=\alpha_{\text {avg }}$, and $\langle\bar{\beta}\rangle=\beta_{\text {avg }}$, and with second moments as in Eqs. (25). If $\bar{\alpha}<0$ set $\bar{\alpha}=0$ and similar for $\bar{\beta}$.

2. Using the $\bar{\alpha}$ and $\bar{\beta}$ just generated, draw independent integer random numbers $m_{A}$ and $m_{B}$ from Poissonian distributions with parameters $\Omega \bar{\alpha} \Delta t$ and $\Omega \bar{\beta} \Delta t$, respectively.

3. For the death processes draw Poissonian random variables $m_{A}^{\prime}$ and $m_{B}^{\prime}$ from Poissonian distributions with parameters $\gamma n_{A} \Delta t$ and $\delta n_{B} \Delta t$ respectively.

4. Update the particle numbers to $n_{A}+m_{A}-m_{A}^{\prime}$ and $n_{B}+m_{B}-m_{B}^{\prime}$, respectively (if this results in $n_{A}<0$ set $n_{A}=0$, and similar for $\left.n_{B}\right)$.

5 . Increment time by $\Delta t$ and go to 1 .

We have introduced a cutoff procedure in step 4, in order to prevent particle numbers from going negative. This is necessary due to the discrete-time nature of the process, and well-known in the context of $\tau$-leaping [46]. In particular this is not related to the appearance of neg- 


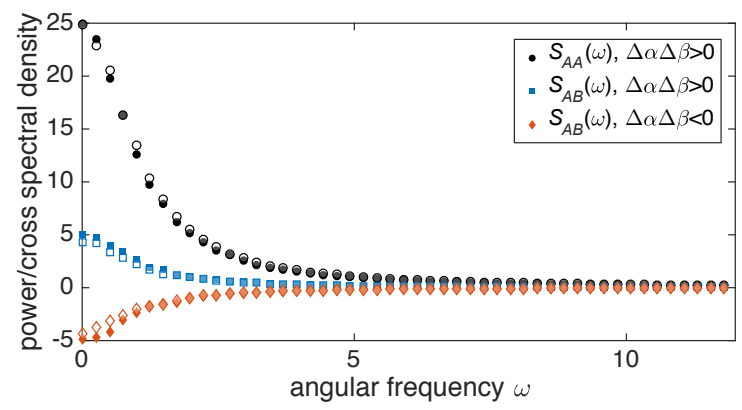

FIG. 6. Spectra of fluctuations from direct simulations of the full model (filled symbols), and using the discrete-time algorithm in Sec. VD (open symbols). The model is the same as in previous figures $\left(k_{0}=k_{1}=1, \Omega=20, \lambda=20, \alpha_{0}=\right.$ $\left.0, \alpha_{1}=1, \Delta t=0.1\right)$.

ative rates in the reduced master equation, and applies in the case $\Delta \alpha \Delta \beta>0$ as well.

We have carried out simulations using this algorithm for both cases $\Delta \alpha \Delta \beta>0$ and $\Delta \alpha \Delta \beta<0$. To test whether the algorithm captures dynamical properties of sample paths we have measured the power spectral density $S_{A A}(\omega)=\left\langle\left|\hat{n}_{A}(\omega)\right|^{2}\right\rangle$, where $\hat{n}_{A}(\omega)$ is the Fourier transform of the random process $n_{A}(t)$. Similarly, we also look at the cross power spectral density $S_{A B}(\omega)=$ $\left\langle n_{A}^{\dagger}(\omega) n_{B}(\omega)\right\rangle$ (the superscript ${ }^{\dagger}$ denotes complex conjugation). These are the Fourier transforms of the autocorrelation and cross-correlation functions respectively.

As shown in Fig. 6 the resulting spectra of fluctuations are in agreement with those of the full model, at least to reasonable approximation. We attribute remaining discrepancies to the discretisation of time and the assumption of Gaussian effective birth rates.

It is important to stress that agreement with the full model requires a careful choice of the time step $\Delta t$. On the one hand, one needs $\Delta t \gtrsim 1 / \lambda$, otherwise it is not justified to replace $\bar{\alpha}$ and $\bar{\beta}$ by Gaussian random variables. On the other hand, the so-called 'leap condition' for $\tau$-leaping must be fulfilled [46], that is, the time step $\Delta t$ must not be long enough for the population to change significantly in one step. More precisely the changes in particle numbers must remain of order $\Omega^{0}$ in each step.

\section{CRITERION FOR THE EMERGENCE OF NEGATIVE RATES}

In the context of the example of Sec. III we have already established that reduced master equations with positive rates describe well-defined Markov processes. If negative rates emerge initial conditions can always be found so that negative solutions result at short times. It is therefore of primary interest to establish what properties of the original system lead to negative rates at the level of the reduced master equation.

In this Section we establish a criterion for the occur- rence of negative rates in equations of the type (14). It covers the class of processes in which the environmental dynamics has two states, and is independent of the state of the population. We also assume that switches of the environmental state do not change the state of the population.

\section{Model and notation}

We look at a general population in which each individual is of one of $S$ species, labelled $i=1, \ldots, S$. We write $n_{i}$ for the number of individuals of species $i$ in the population, and $\mathbf{n}=\left(n_{1}, \ldots, n_{S}\right)$. We label the different reactions that can occur in this system by $r=1,2 \ldots, r_{\text {max }}$. We write $R_{r \sigma}(\mathbf{n})$ for the rate with which reaction $r$ occurs if the population is in state $\mathbf{n}$ and the environment in state $\sigma$.

We also introduce stoichiometric coefficients $\nu_{r, i}$, i.e., if a reaction of type $r$ occurs, the number of particles of species $i$ changes by $\nu_{r, i}$. These coefficients can be positive, negative or zero. We write $\boldsymbol{\nu}_{r}=\left(\nu_{r, 1}, \ldots, \nu_{r, S}\right)$. In other words, if a reaction of type $r$ occurs the state of the population changes from $\mathbf{n}$ to $\mathbf{n}+\boldsymbol{\nu}_{r}$.

The operators $\mathcal{M}_{\sigma}$ are then given by

$$
\mathcal{M}_{\sigma}=\sum_{r}\left(\mathcal{E}^{\boldsymbol{\nu}_{r}}-1\right) R_{r \sigma}(\mathbf{n}),
$$

where we have used the following notation

$$
\mathcal{E}^{\boldsymbol{\nu}} f(\mathbf{n})=f(\mathbf{n}+\boldsymbol{\nu})
$$

that is to say $\mathcal{E}^{\nu}=\prod_{i} \mathcal{E}_{i}^{\nu_{i}}$, where $\mathcal{E}_{i}$ is the creation operator for individuals of species $i$.

\section{Reduced master equation}

The operator on the RHS of the reduced master equation (14) is of the form

$$
\mathcal{M}_{\text {red }}=\mathcal{M}_{\text {avg }}+\frac{1}{2} \frac{\theta^{2}}{\lambda}\left(\mathcal{M}_{0}-\mathcal{M}_{1}\right)^{2},
$$

where $\mathcal{M}_{\text {avg }}=\sum_{r}\left(\mathcal{E}^{\boldsymbol{\nu}_{r}}-1\right) R_{r, \text { avg }}(\mathbf{n})$. After some algebra one finds for the current system,

$\mathcal{M}_{\text {red }}=\sum_{r}\left(\mathcal{E}^{\boldsymbol{\nu}_{r}}-1\right) R_{r, \mathrm{eff}}(\mathbf{n})+\sum_{r q}\left(\mathcal{E}^{\boldsymbol{\nu}_{r}} \mathcal{E}^{\boldsymbol{\nu}_{q}}-1\right) R^{(r q)}(\mathbf{n})$,

where we have

$$
\begin{aligned}
R_{r, \mathrm{eff}}(\mathbf{n})= & R_{r, \text { avg }}(\mathbf{n}) \\
& -\frac{\theta^{2}}{2 \lambda} \Delta_{r}(\mathbf{n}) \sum_{q}\left[\Delta_{q}(\mathbf{n})+\Delta_{q}\left(\mathbf{n}-\boldsymbol{\nu}_{r}\right)\right], \\
R^{(r q)}= & \frac{\theta^{2}}{\lambda} \Delta_{r}\left(\mathbf{n}-\boldsymbol{\nu}_{q}\right) \Delta_{q}(\mathbf{n}),
\end{aligned}
$$


with the shorthand $\Delta_{r}(\mathbf{n})=R_{r, 0}(\mathbf{n})-R_{r, 1}(\mathbf{n})$.

Reaction $r$ occurs in the reduced dynamics with effective rate $R_{r, \text { eff }}(\mathbf{n})$. For large enough $\lambda$, these rates are always positive. Further, one has burst reactions in the reduced dynamics. These are combinations of two original reactions $r$ and $q$, see the second term on the right-hand side of Eq. (31). These reactions occur with rates $R^{(r q)}(\mathbf{n})$. The rates $R^{(r r)}(\mathbf{n})$ are manifestly nonnegative.

Always assuming that $\lambda$ is sufficiently large so that the $R_{r, \text { eff }}(\mathbf{n})$ are all non-negative, we conclude: If all rates $R^{(r q)}(\mathbf{n})$ are non-negative then the reduced master equation describes a well-defined Markov process. If this is not the case, there exist initial conditions so that the solution of the reduced master equation will have negative transients.

The criterion can be simplified further if the reactions rates do not depend on $\mathbf{n}$ (i.e., when different particles do not interact). The reduced master equation then guarantees positivity if and only if all $\Delta_{r}=R_{r, 0}-R_{r, 1}$ have the same sign (in the weak sense, some of the $\left\{\Delta_{r}\right\}$ may be zero). This is the case when there is one environmental state in which all reactions happen with a rate which is equal or higher than in the other environmental state, i.e., if one environmental state speeds up all reactions compared to the other environment (or at least, it does not make them slower).

\section{SUMMARY AND CONCLUSIONS}

In summary, we have studied Markovian stochastic systems with discrete states, coupled to an external environment switching between discrete states. Our analysis focuses on the limit in which the environmental dynamics are fast relative to that of the system, but where the time scale separation is not necessarily infinite. In particular, we have derived reduced dynamics for the open system, capturing next-order corrections to the adiabatic limit.

The reduced master equation shows reactions which are not present in the original dynamics. These are bursting reactions, typically combinations of two individual reactions of the original dynamics. In some cases negative transition 'rates' emerge.

We have demonstrated that that negative (pseudo-) probabilities can arise in the presence negative rates. $\mathrm{Nu}-$ merical integration of the reduced master equation further suggests that these negative transients only occur on time scales shorter than that of the environmental process. The reduced dynamics is obtained by coarse graining the environmental process, and as a consequence it does not resolve the physics of the problem on such fine time scales. Despite the unphysical transients the reduced master equation can be useful to characterise the stationary distribution of the open system. It approximates this stationary distribution better than an adiabatic approach, in which the time-scale separation is assumed to be infinite.
The emergence of bursting reactions can be understood further by looking at the time evolution of individual sample paths of system and environment over a finite time interval. This leads to a discrete-time approximation for the dynamics of the open system. The path of the environment in one time step can be approximated by Gaussian random variables; bursting in the system results from fluctuations of this discrete-time Gaussian process. We have used this approach to propose a simulation method for stochastic systems coupled to a fastswitching environment with two states.

We note that Refs. [8, 10, 16, 23, 24] use the WentzelKramers-Brillouin (WKB) method to calculate stationary distributions of populations with fast environmental dynamics. While some dynamical properties can be derived from this (e.g., mean first-passage times), the method is not in itself a dynamic approach. It does not provide immediate access to two-time objects such as correlation functions. Our approach is different, it reduces the dynamics of the system by integrating out the environment. The result is a dynamical process. For example, we study spectra of fluctuations in Fig. 6, see also Fig. B1 in the Appendix. We also note that the WKB approach is based on a limit of large population size, and that it often requires the existence of fixed points of the limiting deterministic model. In our derivation of the reduced master equation makes we have not made these assumptions.

Negative rates are can also be found in the reduced dynamics for open quantum systems. In this context one starts from a unitary dynamics of system and environment [20, 21]. Upon tracing out the environment a quantum master equation with temporarily negative decay rates can result. We note one potentially important difference between the classical and the quantum cases; the origin of negative rates in open quantum systems is often attributed to a two-way exchange of information between the system and the environment [20, 21]. This mechanism is not available the two-species example we have looked at (Sec. III). Still, the reduced master equation for this model can have negative rates.

Our work provides several starting points for future work. In the companion paper Ref. [31] we build on the reduction technique developed herein by combining it with approximations to the dynamics of the population itself through expansions in the inverse system size. We describe a number of weak-noise expansions which allow for analytical results or more efficient simulation. The construction in Sec. $\mathrm{V}$ and the associated discrete-time simulation algorithm could be extended to more general models with multiple environmental states. This could provide a powerful tool for the simulation of systems coupled to fast external environments. Further, it would be interesting to study in more detail the analogies and differences between the reduced dynamics for open quantum systems and for classical systems coupled to fast environments. As a first step, one might focus on classical systems in which the dynamics of the environment depends 
on the state of the system itself, and try to characterise the information flow between system and environment. A separate further line of research might focus on systems in which the environment takes continuous states (see e.g. Ref. [10, 23, 24]), and on the comparison with the discrete case.

Acknowledgements. P.G.H., Y.T.L., and T.G. thank the Engineering and Physical Sciences Research Council (EPSRC) for funding (PhD studentship, and Grant No. EP/K037145/1). Y.T.L. was supported by the Center for Nonlinear Studies.

Author contributions. All authors contributed to this work equally.
[1] E. Kussell, R. Kishony, N. Q. Balaban, and S. Leibler, Genetics 169, 1807 (2005).

[2] E. Kussell and S. Leibler, Science 309, 2075 (2005).

[3] S. Leibler and E. Kussell, Proc. Natl. Acad. Sci. U.S.A. 107, 13183 (2010).

[4] P. Thomas, N. Popović, and R. Grima, Proc. Natl. Acad. Sci. U.S.A. 111, 6994 (2014).

[5] T. B. Kepler and T. C. Elston, Biophys. J. 81, 3116 (2001).

[6] M. Thattai and A. Van Oudenaarden, Genetics 167, 523 (2004).

[7] P. S. Swain, M. B. Elowitz, and E. D. Siggia, Proc. Natl. Acad. Sci. U.S.A. 99, 12795 (2002).

[8] M. Assaf, E. Roberts, Z. Luthey-Schulten, and N. Goldenfeld, Phys. Rev. Lett. 111, 058102 (2013).

[9] A. Duncan, S. Liao, T. Vejchodskỳ, R. Erban, and R. Grima, Phys. Rev. E 91, 042111 (2015).

[10] M. Assaf, M. Mobilia, and E. Roberts, Phys. Rev. Lett. 111, 238101 (2013).

[11] P. Ashcroft, P. M. Altrock, and T. Galla, J. R. Soc. Interface 11, 20140663 (2014).

[12] K. Wienand, E. Frey, and M. Mobilia, Phys. Rev. Lett. 119, 158301 (2017).

[13] R. West, M. Mobilia, and A. M. Rucklidge, Phys. Rev. E 97, 022406 (2018).

[14] A. J. Black and A. J. McKane, J. Theor. Biol. 267, 85 (2010).

[15] C. Escudero and J. Á. Rodríguez, Phys. Rev. E 77, 011130 (2008).

[16] M. Assaf, A. Kamenev, and B. Meerson, Phys. Rev. E 78, 041123 (2008).

[17] Q. Luo and X. Mao, J. Math. Anal. Appl. 334, 69 (2007).

[18] C. Zhu and G. Yin, J. Math. Anal. Appl. 357, 154 (2009).

[19] H.-P. Breuer and F. Petruccione, The theory of open quantum systems (Oxford University Press, Great Clarendon Street, Oxford, UK, 2002).

[20] H.-P. Breuer, E.-M. Laine, J. Piilo, and B. Vacchini, Rev. Mod. Phys. 88, 021002 (2016).

[21] I. de Vega and D. Alonso, Rev. Mod. Phys. 89, 015001 (2017).
[22] P. C. Bressloff, Phys. Rev. E. 94, 042129 (2016).

[23] M. Assaf, E. Roberts, and Z. Luthey-Schulten, Phys. Rev. Lett. 106, 248102 (2011).

[24] E. Roberts, S. Be'er, C. Bohrer, R. Sharma, and M. Assaf, Phys. Rev. E 92, 062717 (2015).

[25] P. C. Bressloff and J. M. Newby, Phys. Rev. E 89, 042701 (2014).

[26] P. C. Bressloff, Phy. Rev. E 95, 012124 (2017).

[27] P. C. Bressloff, Phys. Rev. E 95, 012138 (2017).

[28] P. C. Bressloff, Phys. Rev. E 95, 012130 (2017).

[29] J. Bowen, A. Acrivos, and A. Oppenheim, Chem. Eng. Sci. 18, 177 (1963).

[30] L. A. Segel and M. Slemrod, SIAM Review 31, 446 (1989).

[31] P. G. Hufton, Y. T. Lin, and T. Galla, submitted simultaneously to Phys. Rev. E. (2019).

[32] W. Ewens, Mathematical Population Genetics 1 (Springer-Verlag, New York, 2004).

[33] A. Traulsen and C. Hauert, "Stochastic evolutionary game dynamics," in Reviews of Nonlinear Dynamics and Complexity (Wiley-VCH Verlag GmbH and Co. KGaA, 2010) pp. 25-61.

[34] J. M. Sancho and M. San Miguel, J. Stat. Phys. 37, 151 (1984).

[35] E. Hernández-García, L. Pesquera, M. A. Rodríguez, and M. San Miguel, J. Stat. Phys. 55, 1027 (1989).

[36] D. T. Gillespie, J. Comp. Phys. 22, 403 (1976).

[37] D. T. Gillespie, J. Chem. Phys. 81, 2340 (1977).

[38] J. Piilo, S. Maniscalco, K. Härkönen, and K.-A. Suominen, Phys. Rev. Lett. 100, 180402 (2008).

[39] H.-P. Breuer and J. Piilo, EPL (Europhysics Letters) 85, 50004 (2009).

[40] A. Suárez, R. Silbey, and I. Oppenheim, J. Chem. Phys. 97, 5101 (1992).

[41] P. Pechukas, Phys. Rev. Lett. 73, 1060 (1994).

[42] S. Gnutzmann and F. Haake, Z. Phys. B 101, 263 (1996).

[43] F. Benatti, R. Floreanini, and M. Piani, Phys. Rev. A 67, 042110 (2003).

[44] P. RF, Physical Review 162, 186 (1967).

[45] H. Risken and H. Vollmer, Z. Phys. B 35, 313 (1979).

[46] D. T. Gillespie, J. Chem. Phys. 115, 1716 (2001).

\section{Appendix A: State-dependent environmental process}

We briefly consider the case in which the transition matrix for the environmental process depends on the state of the system proper, i.e., $A_{\sigma \rightarrow \sigma^{\prime}}=A_{\sigma \rightarrow \sigma^{\prime}}(\ell)$. From Eq. (8) we have

$$
\frac{\mathrm{d}}{\mathrm{d} t} \Pi(\ell, t)=\sum_{\sigma} \mathcal{M}_{\sigma}\left[\rho^{*}(\sigma \mid \ell) \Pi(\ell, t)\right]+\frac{1}{\lambda} \sum_{\sigma} \mathcal{M}_{\sigma} w_{\sigma}(\ell, t),
$$


and from Eq. (9)

$$
\sum_{\sigma^{\prime}} A_{\sigma^{\prime} \rightarrow \sigma}(\ell) w_{\sigma^{\prime}}(\ell, t)=\rho^{*}(\sigma \mid \ell) \sum_{\sigma^{\prime}} \mathcal{M}_{\sigma^{\prime}}\left[\rho^{*}\left(\sigma^{\prime} \mid \ell\right) \Pi(\ell, t)\right]-\mathcal{M}_{\sigma}\left[\rho^{*}(\sigma \mid \ell) \Pi(\ell, t)\right]
$$

\section{Adiabatic limit}

It is useful to define the following operators, acting on functions $f(\ell)$,

$$
\widehat{\mathcal{M}}_{\sigma} f(\ell)=\mathcal{M}_{\sigma}\left[\rho^{*}(\sigma \mid \ell) f(\ell)\right]
$$

where the right-hand side indicates that the operator $\mathcal{M}_{\sigma}$ acts on the object inside the square bracket. In the adiabatic limit one finds [by sending $\lambda \rightarrow \infty$ in Eq. (A1)]

$$
\frac{\mathrm{d}}{\mathrm{d} t} \Pi(\ell, t)=\widehat{\mathcal{M}}_{\mathrm{avg}} \Pi(\ell, t)
$$

where we now have

$$
\mathcal{M}_{\mathrm{avg}}=\sum_{\sigma} \widehat{\mathcal{M}}_{\sigma}
$$

To illustrate the principle we use a population with $n$ individuals of a single species, and a birth reaction with rate $b_{\sigma}(n)$, which may potentially depend on $n$. We then have $\mathcal{M}_{\sigma}=\left[\mathcal{E}^{-1}-1\right] b_{\sigma}(n)$. We find

$$
\mathcal{M}_{\mathrm{avg}} \Pi(n, t)=\left[\mathcal{E}^{-1}-1\right] b_{\mathrm{avg}}(n) \Pi(n, t),
$$

where

$$
b_{\mathrm{avg}}(n)=\sum_{\sigma} \rho^{*}(\sigma \mid n) b_{\sigma}(n)
$$

We note that $b_{\text {avg }}(n)$ carries a dependence on $n$, even when $b_{\sigma}(n)$ is itself independent of $n$.

\section{Next-order contribution}

We focus on the case of two environmental states, with switching rates $A_{1 \rightarrow 0}(\ell)=k_{0}(\ell)$ and $A_{0 \rightarrow 1}(\ell)=k_{1}(\ell)$. In this case we have $\rho^{*}(0 \mid \ell)=k_{0}(\ell) /\left[k_{0}(\ell)+k_{1}(\ell)\right]$, and $\rho^{*}(1 \mid \ell)=k_{1}(\ell) /\left[k_{0}(\ell)+k_{1}(\ell)\right]$. From Eq. (A2) one then finds

$$
w_{0}(\ell, t)=-w_{1}(\ell, t)=\frac{1}{k_{0}(\ell)+k_{1}(\ell)}\left[\rho^{*}(1 \mid \ell) \widehat{\mathcal{M}}_{0}-\rho^{*}(0 \mid \ell) \widehat{\mathcal{M}}_{1}\right] \Pi(\ell, t)
$$

Inserting into Eq. (A1) we have

$$
\frac{\mathrm{d}}{\mathrm{d} t} \Pi(\ell, t)=\mathcal{M}_{\mathrm{avg}} \Pi(\ell)+\frac{1}{\lambda}\left(\mathcal{M}_{0}-\mathcal{M}_{1}\right) \frac{1}{k_{0}(\ell)+k_{1}(\ell)}\left[\rho^{*}(1 \mid \ell) \widehat{\mathcal{M}}_{0}-\rho^{*}(0 \mid \ell) \widehat{\mathcal{M}}_{1}\right] \Pi(\ell, t),
$$

which can be written as

$$
\frac{\mathrm{d}}{\mathrm{d} t} \Pi(\ell, t)=\mathcal{M}_{\mathrm{avg}} \Pi(\ell)+\frac{1}{\lambda}\left[\widehat{\mathcal{M}}_{0} \rho^{*}(0 \mid \ell)^{-1}-\widehat{\mathcal{M}}_{1} \rho^{*}(1 \mid \ell)^{-1}\right] \frac{1}{k_{0}(\ell)+k_{1}(\ell)}\left[\rho^{*}(1 \mid \ell) \widehat{\mathcal{M}}_{0}-\rho^{*}(0 \mid \ell) \widehat{\mathcal{M}}_{1}\right] \Pi(\ell, t)
$$

While this object is quite lengthy, it formally describes the reduced dynamics to sub-leading order in $1 / \lambda$, and can be used for further analysis. 


\section{Appendix B: Continuous-time simulation algorithms for reduced master equations with negative rates}

\section{Path-level simulation}

\section{a. Description of the simulation algorithm}

A stochastic simulation algorithm was discussed in Ref. [38] for non-Markovian jumps in quantum systems. This method simulates processes defined by quantum master equations with temporarily negative decay rates. The central idea is to represent the solution of the master equation by an ensemble of sample paths, which are generated in parallel. In contrast with standard methods $[36,37]$ these paths are correlated with each other.

We have adapted the algorithm to the case of the classical master equation

$$
\frac{\mathrm{d}}{\mathrm{d} t} \Pi(\ell, t)=\sum_{\ell^{\prime}} R_{\ell^{\prime} \rightarrow \ell} \Pi\left(\ell^{\prime}, t\right),
$$

where some of the rates $R_{\ell^{\prime} \rightarrow \ell}$ may be negative. The algorithm uses Eqs. (20) and (21) to convert reactions with negative rates into reactions in the opposite direction, and with positive renormalised rates. In order to do this we need the entries of the probability distribution, $\Pi(\ell)$ and $\Pi\left(\ell^{\prime}\right)$, see Eq. (20). These in turn are estimated from the ensemble of sample paths. In this way, the trajectories are correlated with each other [38, 39].

We index each trajectory individually, so that we can follow the time evolution of each sample path. At each point in time the ensemble is specified by the state of each of the sample paths. We write $N_{\ell}$ for the number of sample paths in state $\ell$. To keep the notation compact we suppress the time dependence of $N_{\ell}$. One has $\sum_{\ell} N_{\ell}=M$ at all times, where $M$ is the size of the ensemble.

Before we detail the algorithm we describe the construction of a matrix $\mathbf{S}$ with elements $S_{\ell \rightarrow \ell^{\prime}}$ which give the rate of a reaction $\ell \rightarrow \ell^{\prime}$ to occur in the ensemble. The matrix is needed frequently in the algorithm, and is constructed as follows: (i) start with $S_{\ell \rightarrow \ell^{\prime}}=0$ for all $\ell, \ell^{\prime}$; (ii) for all reactions $\ell \rightarrow \ell^{\prime}$ with positive rate $R_{\ell \rightarrow \ell^{\prime}}$ increase $S_{\ell \rightarrow \ell^{\prime}}$ by $R_{\ell \rightarrow \ell^{\prime}}$; (iii) for reactions with negative rate $R_{\ell \rightarrow \ell^{\prime}}$ and $N_{\ell^{\prime}}>0$ construct $T_{\ell^{\prime} \rightarrow \ell}$ as in Eq. (20), where $N_{\ell} / N_{\ell^{\prime}}$ is used as a proxy for $\Pi(\ell) / \Pi\left(\ell^{\prime}\right)$. If $N_{\ell^{\prime}}=0$ set $T_{\ell^{\prime} \rightarrow \ell}=0$. Increase $S_{\ell^{\prime} \rightarrow \ell}$ by $T_{\ell^{\prime} \rightarrow \ell}$; (iv) finally, for all pairs $\ell$, $\ell^{\prime}$ multiply $S_{\ell \rightarrow \ell^{\prime}}$ by $N_{\ell}$. For a given master equation (i.e., a given matrix $\mathbf{R}$ ) the matrix $\mathbf{S}$ is a function of the current state of the ensemble, i.e., of the $\left\{N_{\ell}\right\}$. All entries $S_{\ell \rightarrow \ell^{\prime}}\left(\ell \neq \ell^{\prime}\right)$ are non-negative. The diagonal elements are zero. The element $S_{\ell \rightarrow \ell^{\prime}}$ indicates the rate for a reaction $\ell \rightarrow \ell^{\prime}$ to occur, given the current state of the ensemble. One has $S_{\ell \rightarrow \ell^{\prime}}=0$ if no sample path in the ensemble is in state $\ell$. We also note that the total rate for a reaction of any type to happen, $\sum_{\ell \neq \ell^{\prime}} S_{\ell \rightarrow \ell^{\prime}}$, scales linearly with $M$. This guarantees that each time step in the procedure below is of order $M^{-1}$, or in other words, that order $M$ reactions occur per unit time.

The algorithm proceeds as follows:

1. Given the current state of the ensemble compute the matrix $\mathbf{S}$ as described above.

2. Draw a random time increment $\tau$ from an exponential distribution with parameter $s=\sum_{\ell, \ell^{\prime}} S_{\ell \rightarrow \ell^{\prime}}$.

3. Randomly select an origin $\ell$ and a destination $\ell^{\prime}$ with a probability weighted by $S_{\ell \rightarrow \ell^{\prime}}$ (i.e., the probability that $\ell$ is picked as an origin and $\ell^{\prime}$ as a destination is $S_{\ell \rightarrow \ell^{\prime}} / s$ ).

4. Randomly (with equal probabilities) pick one of the sample paths currently in state $\ell$ and change its state to $\ell^{\prime}$.

5. Increment time by $\tau$ and go to step 1 .

We note that this algorithm does not allow for any state $\ell$ to ever have a negative occupancy $N_{\ell}$. Furthermore, if all $R_{\ell \rightarrow \ell^{\prime}}$ are non-negative the simulation reduces to the standard Gillespie algorithm [36, 37]. In this case the sample paths remain uncorrelated.

\section{b. Test of the algorithm}

Here, we show this method can fail to produce sample paths which are representative of the full model. We use the example in Eq. (17). The algorithm captures the stationary distribution accurately, as illustrated by the markers in Fig. $2(\mathrm{~h})$. Next, we test whether the simulation reproduces dynamical properties of the sample paths of the full model. Specifically we focus on the power and cross spectra defined in Sec. VD.

Results are shown in Figure B1. These are measured in the regime when $\Pi\left(n_{A}, n_{B}\right)$ has reached the stationary state, and averaged over a large ensemble of trajectories. 

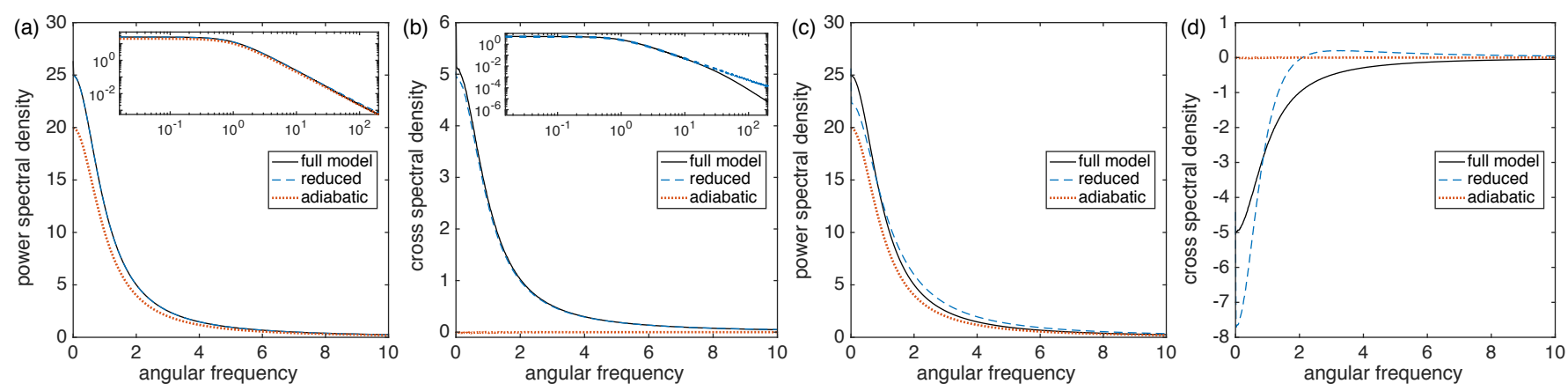

FIG. B1. Spectra of fluctuations for the model defined in Sec. III B. Panels (a) and (b) are for $\Delta \alpha \Delta \beta>0$; (c) and (d) for $\Delta \alpha \Delta \beta<0$. We show the power spectral density $S_{A A}(\omega)$ in (a) and (c), and the cross spectral density $S_{A B}(\omega)$ in (b) and (d); the insets show the same quantities on a logarithmic scale. Parameters: $\alpha_{0}=0, \alpha_{1}=1, \lambda k_{0}=\lambda k_{1}=20, \Omega=20$ in all panels; $\beta_{0}=0, \beta_{1}=1$ in $(\mathrm{a}, \mathrm{b}) ; \beta_{0}=1, \beta_{1}=0$ in $(\mathrm{c}, \mathrm{d})$.

Only positive rates in the reduced master equation. Panels (a) and (b) serve as a benchmark, and show the case $\Delta \alpha \Delta \beta>0$ when all rates in the reduced master equation are positive. The above simulation scheme then reduces to the standard Gillespie method. As seen in the figure the power and cross spectra $S_{A A}(\omega)$ and $S_{A B}(\omega)$ obtained from simulating paths of the reduced master equation agree well with those from simulations of the full model, at least at sufficiently low frequencies $\omega$. At larger frequencies deviations are seen, this is particularly visible for the cross spectrum; see the inset of panel (b). These deviations between reduced and the full model are not surprising; the reduced model does not resolve the mechanics of the environment on short time scales. Spectra obtained from sample paths of the master equation in the adiabatic limit show significant deviations from those of the full model; we note in particular that the cross spectrum $S_{A B}(\omega)$ vanishes [dotted red line in Fig. B1 (b)].

Negative rates. Results for the case with negative rates in the reduced master equation are shown in panels (c) and (d) of Fig. B1. We find marked differences between the spectra generated from the reduced master equation with the above algorithm and those of sample paths of the full model. This is particularly noticeable in the cross spectrum in panel (d), which is strictly negative in the full model, but comes out positive at intermediate frequencies if the above simulation method is used for the reduced dynamics.

We conclude that the trajectories generated by the simulation algorithm in Appendix B 1 a do not represent sample paths of the full model when the reduced master equation contains negative rates. Our findings invite the question whether algorithms of this type [38, 39] provide a faithful representation of the full dynamics of open quantum systems and their environment.

\section{Distribution-level simulation}

The time-dependent solution $\Pi(\ell, t)$ can be obtained by direct numerical integration of the reduced master equation, for example using a Runge-Kutta scheme. However for large state spaces this approach can become slow. The technique described in this Section can, in some cases, provide a faster alternative.

We consider a large number $M$ of discrete units of probability, $1 / M$. At each point in time the state of the simulation is defined by the 'occupation numbers' $N_{\ell}$ for all states $\ell$; some of the $N_{\ell}$ may be negative. One has $\sum_{\ell} N_{\ell}=M$.

The algorithm proceeds along the following steps:

1. For given occupation numbers $N_{\ell}$ at time $t$, make a list of all possible reactions, labelled by index $\gamma$. Each reaction has a site of origin, $\ell_{\gamma}$, a destination site, $\ell_{\gamma}^{\prime}$, and rate $r_{\gamma}=R_{\ell_{\gamma} \rightarrow \ell_{\gamma}^{\prime}} N_{\ell_{\gamma}}$. Some of the $r_{\gamma}$ may be negative.

2. Draw a random number $\tau$ from an exponential distribution with parameter $\sum_{\gamma}\left|r_{\gamma}\right|$.

3. Pick a reaction from the list created in 1 . The probability to pick $\gamma$ is $\left|r_{\gamma}\right| / \sum_{\gamma^{\prime}}\left|r_{\gamma^{\prime}}\right|$.

4. If $r_{\gamma}>0$ increase $N_{\ell_{\gamma}^{\prime}}$ by one and reduce $N_{\ell_{\gamma}}$ by one. If $r_{\gamma}<0$ reduce $N_{\ell_{\gamma}^{\prime}}$ by one and increase $N_{\ell_{\gamma}}$ by one.

5 . Increment time by $\tau$, and go to 1 .

The process in step 4 allows occupation numbers to go negative. The typical time step of this scheme is given by $1 / \sum_{\gamma}\left|r_{\gamma}\right|$, and reaction $\gamma$ is triggered with probability $\left|r_{\gamma}\right| /\left(\sum_{\gamma^{\prime}}\left|r_{\gamma^{\prime}}\right|\right)$. Thus $\left|r_{\gamma}\right|$ reactions of type $\gamma$ are triggered 
per unit time. The sign convention in step 4 ensures correct sampling of the reduced master equation.

We tested this procedure for the example given by Eq. (17). Results are shown in Fig. 3; there is near perfect agreement between the Monte Carlo procedure and direct numerical integration of the reduced master equation. We stress that this algorithm does not generate sample paths for the reduced master equation. 